\title{
The influence of timing of coronary angiography on acute kidney injury in out-of-hospital cardiac arrest patients: a retrospective cohort study
}

Gladys N. Janssens ${ }^{1}$, Joost Daemen², Jorrit S. Lemkes ${ }^{1}$, Eva M. Spoormans ${ }^{1}$, Dieuwertje Janssen ${ }^{1}$ Corstiaan A. den Uil ${ }^{2,3,4}$, Lucia S. D. Jewbali², Ton A. C. M. Heestermans ${ }^{5}$, Victor A. W. M. Umans ${ }^{5}$, Frank R. Halfwerk ${ }^{6}$, Albertus Beishuizen ${ }^{7}$, Joris Nas ${ }^{8}$, Judith Bonnes ${ }^{9}$, Peter M. van de Ven ${ }^{9}$, Albert C. van Rossum ${ }^{1}$, Paul W. G. Elbers ${ }^{10}$ and Niels van Royen ${ }^{1,8^{*}}$ (B)

\begin{abstract}
Background: Acute kidney injury (AKI) is a frequent complication in cardiac arrest survivors and associated with adverse outcome. It remains unclear whether the incidence of AKI increases after the post-cardiac arrest contrast administration for coronary angiography and whether this depends on timing of angiography. Aim of this study was to investigate whether early angiography is associated with increased development of AKI compared to deferred angiography in out-of-hospital cardiac arrest (OHCA) survivors.

Methods: In this retrospective multicenter cohort study, we investigated whether early angiography (within $2 \mathrm{~h}$ ) after OHCA was non-inferior to deferred angiography regarding the development of AKI. We used an absolute difference of $5 \%$ as the non-inferiority margin. Primary non-inferiority analysis was done by calculating the risk difference with its $90 \%$ confidence interval $(\mathrm{Cl})$ using a generalized linear model for a binary outcome. As a sensitivity analysis, we repeated the primary analysis using propensity score matching. A multivariable model was built to identify predictors of acute kidney injury.

Results: A total of 2375 patients were included from 2009 until 2018, of which 1148 patients were treated with early coronary angiography and 1227 patients with delayed or no angiography. In the early angiography group 18.5\% of patients developed AKI after OHCA and $24.1 \%$ in the deferred angiography group. Risk difference was - 3.7\% with $90 \% \mathrm{Cl}$ ranging from -6.7 to $-0.7 \%$, indicating non-inferiority of early angiography. The sensitivity analysis using propensity score matching showed accordant results, but no longer non-inferiority of early angiography. The factors time to return of spontaneous circulation (odds ratio [OR] 1.12, 95\% Cl 1.06-1.19, $p<0.001$ ), the (not) use of angiotensin-converting enzyme inhibitor or angiotensin II receptor blocker (OR $0.20,95 \% \mathrm{Cl} 0.04-0.91, p=0.04$ ) and baseline creatinine $(\mathrm{OR} 1.05,95 \% \mathrm{Cl} 1.03-1.07, p<0.001)$ were found to be independently associated with the development of AKI.
\end{abstract}

*Correspondence: niels.vanroyen@radboudumc.nl

${ }^{1}$ Department of Cardiology, Amsterdam Cardiovascular Sciences, Amsterdam UMC, Vrije Universiteit Amsterdam, De Boelelaan 1117,

1081HV Amsterdam, The Netherlands

Full list of author information is available at the end of the article

\section{Springer Open}

(c) The Author(s) 2022. Open Access This article is licensed under a Creative Commons Attribution 4.0 International License, which permits use, sharing, adaptation, distribution and reproduction in any medium or format, as long as you give appropriate credit to the original author(s) and the source, provide a link to the Creative Commons licence, and indicate if changes were made. The images or other third party material in this article are included in the article's Creative Commons licence, unless indicated otherwise in a credit line to the material. If material is not included in the article's Creative Commons licence and your intended use is not permitted by statutory regulation or exceeds the permitted use, you will need to obtain permission directly from the copyright holder. To view a copy of this licence, visit http://creativecommons.org/licenses/by/4.0/. 
Conclusions: Although AKI occurred in approximately 20\% of OHCA patients, we found that early angiography was not associated with a higher AKI incidence than a deferred angiography strategy. The present results implicate that it is safe to perform early coronary angiography with respect to the risk of developing AKI after OHCA.

Keywords: Acute kidney injury, Out-of-hospital cardiac arrest, Coronary angiography, Creatinine, Risk factors, Reperfusion injury

\section{Background}

In patients who achieve return of spontaneous circulation (ROSC) after out-of-hospital cardiac arrest (OHCA), the subsequent high morbidity and mortality are mostly due to neurologic injury, systemic ischemia-reperfusion injury and multi-organ dysfunction. Although postresuscitation treatment for OHCA patients has improved over the years, prognosis for these patients remains poor. Several key predictors for survival after the event have been identified, and include witnessed arrest, early initiation of cardiopulmonary resuscitation, time to ROSC and control of acute kidney injury (AKI) [1]. AKI is often observed after cardiac arrest and associated with the occurrence of chronic kidney disease and adverse outcome [2-6]. Whether cardiac arrest patients develop AKI depends on several factors such as age, comorbidity, prior renal insufficiency and time to ROSC $[5,7]$. Other risk factors include diabetes and hypertension [8-10]. Congestive heart failure, hypotension after cardiac arrest, female sex and the usage of an intra-aortic balloon pump are also associated with increased incidence of AKI [9].

Another factor that might influence the development of AKI after cardiac arrest is the usage of iodinated contrast during imaging techniques such as coronary angiography [11]. Currently, a delayed as opposed to immediate angiography is recommended to be considered in OHCA patients without ST-elevation. In the decision process when to perform angiography, the absence of an increased AKI incidence would permit both strategies. At present, it is unclear whether the incidence of AKI increases after the administration of iodinated contrast agents post-cardiac arrest and whether the timing of administration influences the development of AKI. Therefore, the main goal of this study was to test the hypothesis that in patients who are successfully resuscitated after cardiac arrest, a strategy of early coronary angiography would not be worse than a strategy of deferred angiography with respect to AKI.

\section{Methods}

\section{Study design}

Using data from 19 of 23 Dutch cardiac intervention centers, a retrospective cohort study of patients resuscitated after OHCA between January 2009 and July 2018 was conducted. The study protocol was approved by the IRB of the VU University Medical Center and ethics approval was obtained by the medical ethics committee of the VU University Medical Center, Amsterdam, the Netherlands, with approval number 2017.398. Informed consent was waived, as it was not achievable to obtain this from all patients of the large retrospective study cohort.

\section{Patients}

Patients admitted to the participating hospitals and coded with OHCA were screened for inclusion in the study. Patients were eligible for this study if they were 18 years or older and had ROSC. Patients were excluded when they had no creatinine values until day 3 or if time from OHCA to angiography was unknown.

\section{Treatment}

Post-resuscitation care was at the discretion of the clinicians responsible for the patient. According to international guidelines, in patients with persistent ST-elevation on the electrocardiogram (ECG) emergency angiography and subsequent percutaneous coronary intervention (PCI) was performed [12, 13]. In patients without ST-elevation, angiography was either performed immediately or delayed $[14,15]$. Patients receiving angiography within $2 \mathrm{~h}$ after OHCA were considered to have had early angiography.

\section{Data collection and outcomes}

Patient data were anonymized by assigning a random record number to each patient included in the study. Information regarding demographics, pre-hospital settings, status at admission, medical history, clinical and laboratory data (e.g., renal function, blood cell count, $\mathrm{pH}$, lactate) was collected from patient records. Also, available renal function tests at 1 month and 1 year were collected.

The primary endpoint of this study was the AKI incidence in patients receiving early angiography compared to in patients with delayed or no angiography. AKI was defined according to the well-established Acute Kidney Injury Network (AKIN) criteria, ranging from stage 1 to 3 , with higher stages indicating more severe renal failure (see Additional file 1) [16-18]. We used the creatinine level at admission as baseline value. Daily 
serum creatinine was measured in local laboratories. The highest creatinine value within $48 \mathrm{~h}$ after hospital admission was used to calculate the AKIN category to evaluate the effect of OHCA and, if performed, early angiography on kidney function. Because urine output per day but not per hour was collected, we used these additional data to classify AKIN in an additional analysis.

\section{Statistical analysis}

Minimal sample size was calculated for the expected incidence and difference of AKI between the early and deferred angiography group. The previous study by Petek et al. showed an overall incidence of AKI of $15 \%$ with a (non-significant) difference of $4.3 \%$ between early and non-early angiography [19]. With an expected AKI incidence of $20 \%$ in both groups and a non-inferiority margin of $5 \%$ for the risk difference, we would need 792 patients per treatment group to have $80 \%$ power assuming noninferiority testing at a one-sided significance level of 5\%.

Primary non-inferiority analysis was done by calculating the risk difference with its $90 \%$ confidence interval (CI) using a generalized linear model for a binary outcome and the identity link-function. As a sensitivity analysis, we repeated the primary analysis using propensity score matching. Patients who underwent early angiography were matched with patients with deferred or no angiography, according to their propensity score. The propensity score was defined as the probability that a patient would be assigned to a treatment (early or deferred angiography) based upon the clinically plausible explanatory variables including age, sex, comorbidity (i.e., diabetes mellitus, chronic kidney disease, hypertension), serum creatinine level, witnessed cardiac arrest, Glasgow Coma Scale $<8$, first rhythm and signs of STelevation myocardial infarction (STEMI) on the ECG. Propensity scores for early vs. delayed or no angiography were estimated using a match tolerance of 0.1 .

Continuous variables were compared using the independent-samples $t$-test for normally distributed data and summarized by mean and standard deviation. Skewed data were compared using the Mann-Whitney $U$ test and summarized by median and interquartile range. Categorical variables were compared using the Chi-square test or Fisher's exact test in case of low expected cell counts and summarized by counts and percentages.

Univariable and multivariable logistic regression analysis was used to identify predictors of AKI. Statistical significance was assumed when $p$-values for two-sided testing were $<0.05$, except for the primary non-inferiority hypothesis for which a $p$-value for one-sided testing of
$<0.05$ was considered significant. Further details of the statistical analysis are provided in Additional file 1.

\section{Results \\ Patients}

A total of 2375 OHCA patients were included in this study. Of these patients 1148 were treated with early angiography and 1227 with delayed or no angiography (Additional file 1: Fig. S1). Most patients were male $(69.6 \%)$ (Table 1). Patients in the deferred angiography group were slightly older than patients in the early angiography group $(62.7 \pm 12.5$ vs. $64.0 \pm 14.9, p=0.02)$. In both groups ventricular tachycardia/ventricular fibrillation was the most common arrest rhythm, but more frequently in the early angiography group $(93.2 \%$ vs. $75.5 \%$, $p<0.001)$. Overall, the median time to ROSC was $15 \mathrm{~min}$ (10-23). Patients in the deferred angiography group were more frequently comatose on admission $(92.1 \%$ vs. $86.9 \%$, $p=0.001$ ), while patients in the early angiography group more often had a STEMI [12] at medical presentation $(42.3 \%$ vs. $13.4 \%, p<0.001)$. In the deferred angiography group, more patients had chronic kidney disease $(8.8 \%$ vs. $3.4 \%, p<0.001)$ and accordingly their median creatinine values at admission were higher than in the early angiography group (Table 1, Fig. 1, Additional file 1: Table S1). A comparison of baseline characteristics of the matched patients can be found in Additional file 1: Table S2.

\section{Treatments}

Angiography was performed in $48.9 \%$ of patients with deferred angiography (Table 2). Median time to angiography was 75 (52-93) minutes in the early angiography group and 1003 (151-8795) minutes in the delayed angiography group $(p<0.001)$, with a maximum time frame depending on length of intensive care unit stay. PCI was performed in $82.7 \%$ of the patients in the early angiography group and in $36.2 \%$ of the deferred angiography group $(p<0.001)$. Furthermore, patients in the early angiography group underwent a computerized tomography (CT) scan more frequently than patients in the deferred angiography group $(54.2 \%$ vs. $30.8 \%, p<0.001)$. Approximately $80 \%$ of patients in both groups were treated with targeted temperature management.

\section{Acute kidney injury}

In the early angiography group $18.5 \%$ of patients and in the deferred angiography group $24.1 \%$ developed AKI after OHCA $(p=0.002)$. Risk difference was $-3.7 \%$ with $90 \% \mathrm{CI}-6.7$ to $-0.7 \%$, indicating non-inferiority of early angiography in terms of AKI after OHCA. When using urine output for defining AKI as well, this revealed higher proportions of AKI in both groups with the same trend favoring early angiography (early angiography $30.2 \%$ vs. 
Table 1 Baseline characteristics

\begin{tabular}{|c|c|c|c|c|}
\hline Characteristics & All patients $(N=2375)$ & Early CAG $(N=1148)$ & $\begin{array}{l}\text { Deferred/no CAG } \\
(N=1227)\end{array}$ & $P$ value \\
\hline \multicolumn{5}{|l|}{ Patient characteristics } \\
\hline Male sex & 1652 (69.6) & $791(47.9)$ & $861(52.1)$ & 0.50 \\
\hline Age in years & $63.3 \pm 13.8$ & $62.7 \pm 12.5$ & $64.0 \pm 14.9$ & 0.02 \\
\hline Hypertension & $839 / 2041(41.1)$ & $411 / 966(42.5)$ & $428 / 1075$ (39.8) & 0.21 \\
\hline Diabetes mellitus & $379 / 2219(17.1)$ & $180 / 1109(16.2)$ & 199/1110 (17.9) & 0.29 \\
\hline Hypercholesterolemia & 288/1211 (23.8) & $128 / 530(24.2)$ & $160 / 681(23.5)$ & 0.88 \\
\hline Previous cardiac arrest & 21/1003 (2.1) & $9 / 577(1.6)$ & $12 / 426(2.8)$ & 0.17 \\
\hline Previous myocardial infarction & $495 / 2189(22.6)$ & $231 / 1100(21.0)$ & $264 / 1089(24.2)$ & 0.07 \\
\hline Previous CVA or TIA & $43 / 690(6.2)$ & $24 / 357(6.7)$ & 19/333 (5.7) & 0.64 \\
\hline Chronic kidney disease & $69 / 1141(6.0)$ & $20 / 586(3.4)$ & 49/555 (8.8) & $<0.001$ \\
\hline Previous renal replacement therapy & $15 / 1009(1.5)$ & $7 / 579(1.2)$ & $8 / 430(1.9)$ & 0.40 \\
\hline \multicolumn{5}{|l|}{ Pre-hospital characteristics } \\
\hline Arrest witnessed & $981 / 1227(80.0)$ & 495/608 (81.4) & $486 / 619(78.5)$ & 0.20 \\
\hline First rhythm & & & & $<0.001$ \\
\hline VFNT & 1009/1193 (84.6) & $572 / 614(93.2)$ & $437 / 579(75.5)$ & \\
\hline PEA & $57 / 1193(4.8)$ & $12 / 614(2.0)$ & $45 / 579(7.8)$ & \\
\hline Asystole & 127/1193 (10.6) & 30/614 (4.9) & $97 / 579(16.8)$ & \\
\hline Time from arrest to BLS in minutes & $3[1-5]$ & $3[1-5]$ & $3[1-5]$ & 0.40 \\
\hline Time from arrest to ROSC in minutes & 15 [10-23] & 15 [10-23] & 15 [10-23] & 0.69 \\
\hline \multicolumn{5}{|l|}{ Characteristics on hospital arrival } \\
\hline Glasgow Coma Scale $<8$ & 1374/1533 (89.6) & 639/735 (86.9) & 735/798 (92.1) & 0.001 \\
\hline Signs of STEMI on ECG & $543 / 1965(27.6)$ & $410 / 969(42.3)$ & 133/996 (13.4) & $<0.001$ \\
\hline CT scan performed & $320 / 751(57.4)$ & 205/378 (54.2) & 115/373 (30.8) & $<0.001$ \\
\hline \multicolumn{5}{|l|}{ Laboratory values upon admission } \\
\hline $\mathrm{pH}$ & $7.2[7.1-7.3]$ & $7.2[7.1-7.3]$ & $7.2[7.1-7.3]$ & 0.05 \\
\hline Lactate, $\mathrm{mmol} / \mathrm{L}$ & $5.9[3.5-9.1]$ & $5.5[3.5-8.8]$ & $6.2[3.7-9.4]$ & 0.01 \\
\hline Hemoglobin, mmol/L & $8.4 \pm 1.3$ & $8.5 \pm 1.2$ & $8.3 \pm 1.3$ & 0.001 \\
\hline Hematocrit & $0.40 \pm 0.06$ & $0.41 \pm 0.1$ & $0.39 \pm 0.1$ & 0.001 \\
\hline Leukocytes, $\cdot 10^{9} / \mathrm{L}$ & $12.5[9.8-16.7]$ & $12.6[10.0-17.0]$ & $12.2[9.4-16.1]$ & 0.07 \\
\hline $\mathrm{CRP}, \mathrm{mg} / \mathrm{L}$ & $5[3-17]$ & $4[3-16]$ & $5[3-19]$ & 0.15 \\
\hline Creatinine, $\mu \mathrm{mol} / \mathrm{L}$ & $98[82-117]$ & $96[81-114]$ & $100[82-120]$ & 0.001 \\
\hline Creatinine $>130 \mu \mathrm{mol} / \mathrm{L}$ & $366 / 2342(15.6)$ & 148/1134 (12.9) & 218/1208 (17.8) & 0.001 \\
\hline
\end{tabular}

All data are expressed in proportions of the population with known data and percentages (\%). Plus-minus ( \pm ) values are classified as mean and standard deviation (SD). Brackets are classified as median and interquartile ranges (IQR)

CAG coronary angiography, CVA cerebrovascular accident, TIA transient ischemic attack, VF ventricular fibrillation, $V T$ ventricular tachycardia, $P E A$ pulseless electrical activity, $B L S$ basic life support, ROSC return of spontaneous circulation, STEMI ST-segment elevation myocardial infarction, $C T$ computed tomography, CRP C-reactive protein

deferred angiography $36.5 \%, p=0.002$ ) (Additional file 1: Table S3). After propensity score matching, the proportion that developed AKI after OHCA was $24.6 \%$ in the early angiography group and $27.5 \%$ in the deferred angiography group $(p=0.61)$. Risk difference was $-3.3 \%$ with $90 \%$ CI ranging from -12.8 to $6.3 \%$, favoring early angiography, but no longer indicating non-inferiority of early angiography at the prespecified margin of 5\% (Table 3). The composite endpoint of AKI and mortality within $48 \mathrm{~h}$ showed similar results (early angiography $25.5 \%$ vs. $32.2 \%$ in delayed angiography, $p=0.001$ ). Most frequently patients developed AKIN stage $1(10.8 \%$ in early angiography vs. $13.8 \%$ in the deferred angiography group, $p=0.02$ ) (Fig. 2, Additional file 1: Table S4). Overall, 3.8\% of patients developed AKI stage 2 and 5.3\% AKI stage 3 . Propensity matched analysis showed that the distribution in AKI stages did not differ (Table 3). In both groups, in patients who developed AKI, this occurred most frequently already at day 1 (early angiography group $69.4 \%$ and deferred angiography group $73.6 \%$ ). Within the deferred angiography group, patients without angiography developed more AKI than patients undergoing late 


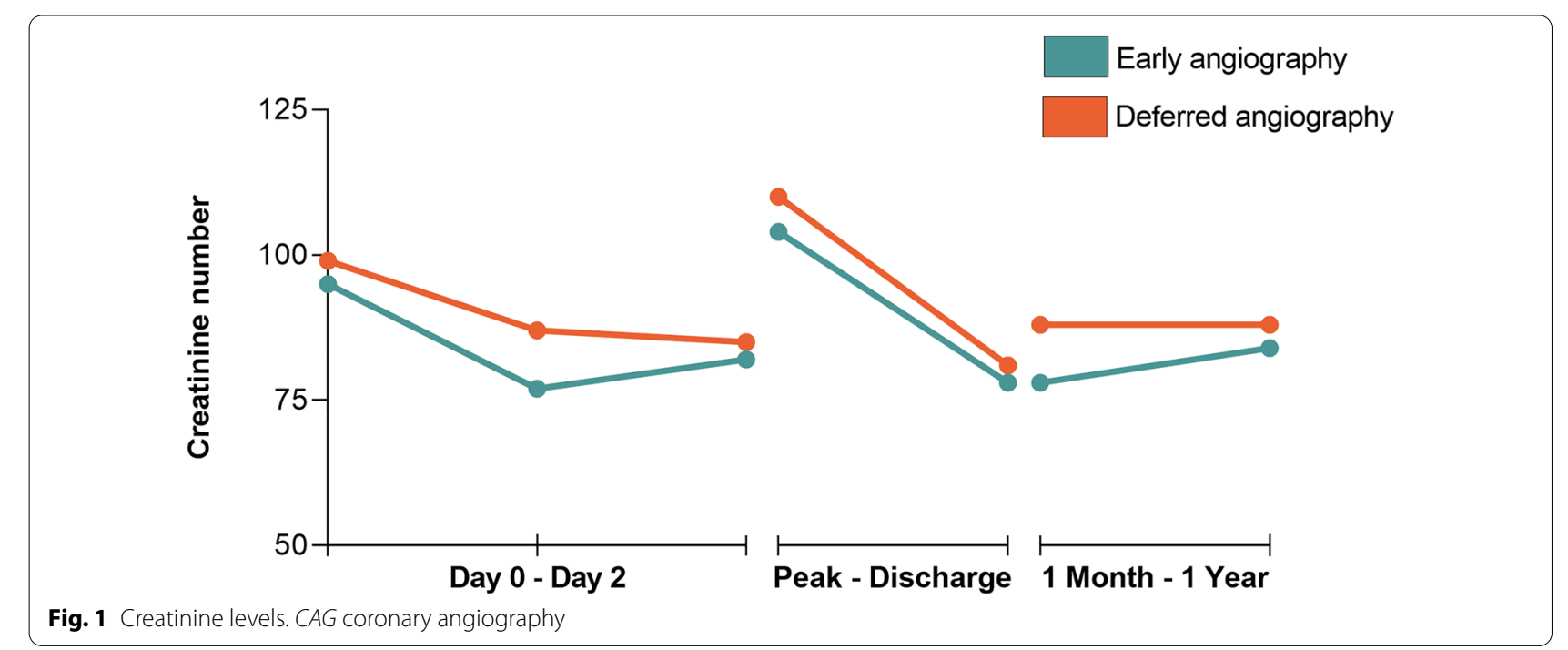

Table 2 In-hospital procedures, treatments and characteristics

\begin{tabular}{|c|c|c|c|}
\hline & Early CAG group $(N=1148)$ & $\begin{array}{l}\text { Deferred CAG/no CAG group } \\
(N=1227)\end{array}$ & $p$-value \\
\hline Targeted temperature management & $670 / 849(78.9)$ & $570 / 709(80.4)$ & 0.47 \\
\hline Hypotension & $244 / 554(44.0)$ & 293/692 (42.3) & 0.55 \\
\hline Use of inotropic or vasopressors & $319 / 512(62.3)$ & $422 / 688(61.3)$ & 0.73 \\
\hline Use of intra-aortic balloon pump & 96/993 (9.7) & 40/1026 (3.9) & $<0.001$ \\
\hline Heart failure $<45 \%$ & 200/496 (40.3) & $134 / 313(42.8)$ & 0.48 \\
\hline Need for renal replacement therapy & $34 / 839(4.1)$ & $42 / 701(6.0)$ & 0.08 \\
\hline Major bleeding ${ }^{a}$ & $15 / 347(4.3)$ & $23 / 357(6.4)$ & 0.21 \\
\hline CAG performed & $1148(100.0)$ & $600 / 1227(48.9)$ & $<0.001$ \\
\hline $\mathrm{PCl}$ performed & 205/248 (82.7) & $129 / 356(36.2)$ & $<0.001$ \\
\hline CABG & $8 / 292(2.7)$ & $9 / 392(2.3)$ & 0.71 \\
\hline \multicolumn{4}{|l|}{ Laboratory values } \\
\hline Lowest pH & $7.2[7.1-7.3]$ & $7.2[7.0-7.3]$ & 0.003 \\
\hline Peak lactate, $\mathrm{mmol} / \mathrm{L}$ & $5.5[3.4-9.1]$ & $6.0[3.4-9.5]$ & 0.38 \\
\hline Peak CRP, mg/L & $105[30-201]$ & $122[61-200]$ & 0.06 \\
\hline Peak leukocytes, $\cdot 10^{9} / \mathrm{L}$ & $18.4[14.3-24.2]$ & $17.6[13.9-23.2]$ & 0.34 \\
\hline Peak CK, U/L & 1604 [629-4421] & $828[296-2411]$ & $<0.001$ \\
\hline Survival at hospital discharge & $544 / 765(71.1)$ & $596 / 850(70.1)$ & 0.66 \\
\hline
\end{tabular}

All data are expressed in proportions of the population with known data and percentages (\%). Brackets are classified as median and interquartile ranges (IQR) CAG coronary angiography, $P C l$ percutaneous coronary intervention, $C A B G$ coronary artery bypass graft, CRP C-reactive protein, $C K$ creatine kinase

${ }^{\text {a }}$ Major bleeding was scored using BARC classification scores $\geq 3$, ranging from bleeding that is not actionable to fatal bleedings

angiography ( $24.4 \%$ vs. $18.8 \%, p=0.037)$. Renal replacement therapy (RRT) was not performed more frequently in the early angiography group ( $4.1 \%$ vs. $6.0 \%, p=0.08)$ (Table 2). Because RRT could have been used for other reasons than kidney failure, we evaluated AKI (stage 3) without the application of RRT as a criterion. Twentyone patients had no AKI anymore after reclassifying. It did not significantly alter the outcomes (Additional file 1:
Table S5). Patients undergoing a CT scan (with the use of contrast) did not develop AKI more frequently (Additional file 1: Table S6). Creatinine at day 3 was persistently higher in the deferred angiography group compared to in patients with early angiography $(84[67-126] \mu \mathrm{mol} / \mathrm{L}$ vs. 79 [65-102] $\mu \mathrm{mol} / \mathrm{L}, p<0.001$ ) (Fig. 1, Additional file 1: Table S1). Creatinine before discharge and available 
Table 3 Sensitivity analysis of acute kidney injury outcomes in matched patient cohorts

\begin{tabular}{lccc}
\hline AKI outcome & Early CAG $(\boldsymbol{n}=\mathbf{1 2 5})$ & $\begin{array}{l}\text { Deferred/no } \\
\text { CAG }(\boldsymbol{n}=\mathbf{1 2 5})\end{array}$ & $\boldsymbol{p}$-value \\
\hline AKI present & $29 / 118(24.6)$ & $33 / 120(27.5)$ & 0.61 \\
AKI stage & & & 0.51 \\
$\quad$ No AKI & $89 / 118(75.4)$ & $87 / 120(72.5)$ & \\
1 & $18 / 118(15.3)$ & $16 / 120(13.3)$ & \\
2 & $3 / 118(2.5)$ & $8 / 120(6.7)$ & \\
3 & $8 / 118(6.8)$ & $9 / 120(7.5)$ & \\
Survival at hospi- & $84 / 125(67.2)$ & $84 / 125(65.2)$ & 1.00 \\
tal discharge & & & \\
\hline
\end{tabular}

All data are expressed in proportions of the population with known data and percentages (\%)

$A K l$ acute kidney injury, CAG coronary angiography

creatinine values 1 month and 1 year after discharge were not different between the groups.

Patients who developed AKI were characterized by older age, a greater proportion of diabetes, a lower incidence of witnessed arrest and shockable rhythm, a longer duration from arrest to basic life support and ROSC, more frequently hypotension, heart failure and treatment with inotropics or vasopressors and targeted temperature management (Additional file 1: Table S7).

Logistic regression analysis was performed to identify predictors of AKI (Table 4). Time to ROSC (odds ratio [OR] 1.12, 95\% CI 1.06-1.19, $p<0.001$ ), the (not) use of an angiotensin-converting enzyme inhibitor (ACEI) or angiotensin II receptor blocker (ARB) (OR 0.20, 95\% CI 0.04-0.91, $p=0.04$ ) and baseline creatinine (OR 1.05, 95\% CI 1.03-1.07, $p<0.001)$ were identified as independent predictors for AKI. After correction for risk factors, early coronary angiography and amount of contrast were not found to be associated with the occurrence of AKI.

\section{Mortality}

Mortality within $48 \mathrm{~h}$ was $7.5 \%$ in the early angiography group and $11.2 \%$ in the deferred group. Survival until discharge was relatively high at $70.6 \%$ and did not differ between the groups, neither after propensity score matching (Tables 2 and 3). In patients with AKI stage 3 , survival at discharge and 1 year was lower at $44.4 \%$, respectively, $31.0 \%$, both $p<0.001$. Of the patients treated with RRT $47.0 \%$ survived until discharge $(p=0.006)$ and $38.6 \%$ were still alive after 1 year.

\section{Discussion \\ Key findings}

In OHCA patients, early angiography did not show a higher incidence or grade of AKI. These findings suggest that coronary angiography can be performed safely either early or delayed in patients after OHCA with regard to the development of AKI. This outcome is an important addition to the results of the randomized COACT trial, which showed a similar survival with immediate and delayed angiography in OHCA patients without STEMI

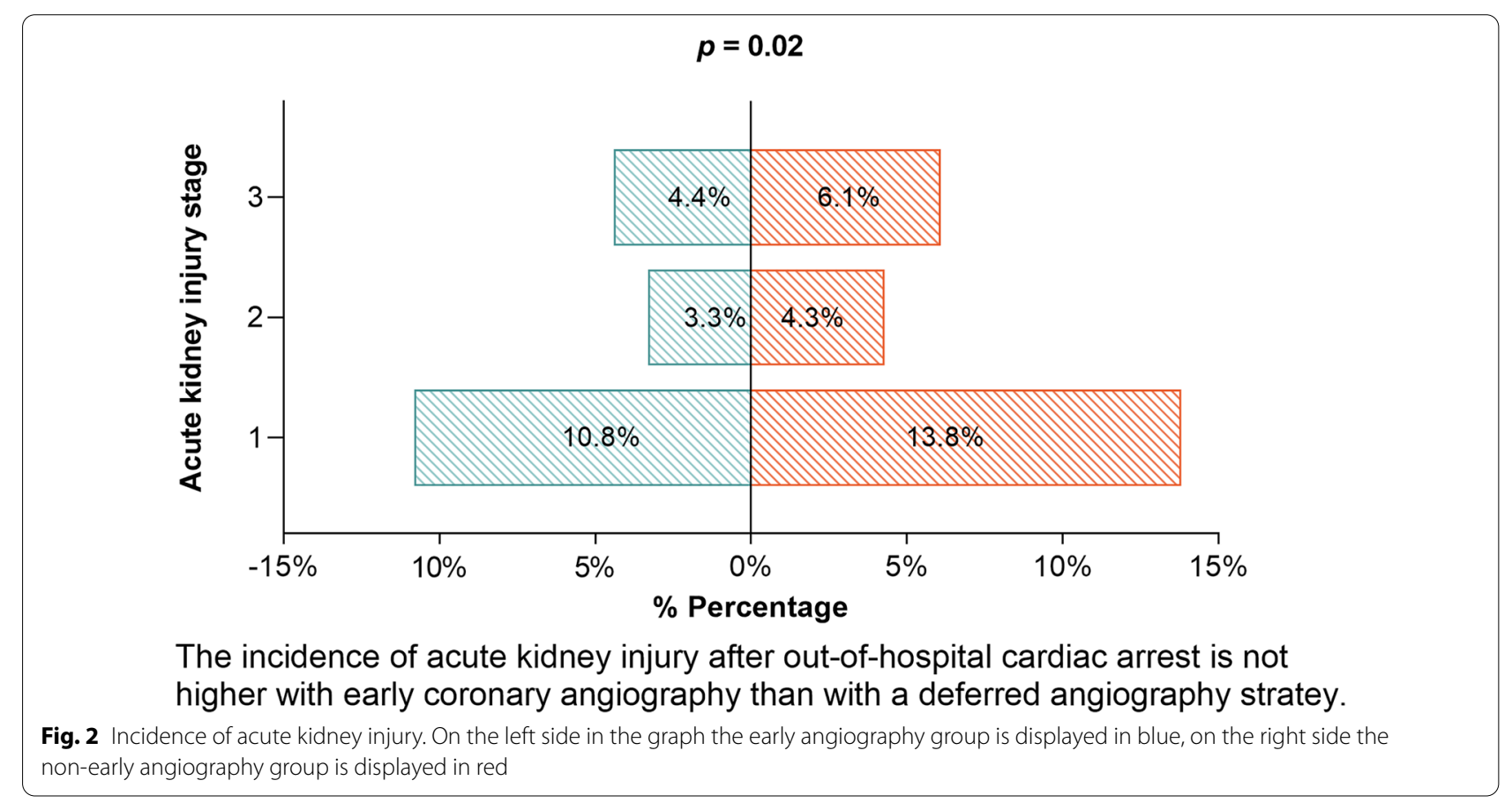


Table 4 Univariable and multivariable analysis on predictors of acute kidney injury

\begin{tabular}{|c|c|c|c|c|}
\hline \multirow[t]{2}{*}{ Predictors and covariates } & \multicolumn{2}{|l|}{ Univariable analysis } & \multicolumn{2}{|c|}{ Multivariable analysis* } \\
\hline & Odds ratio $(95 \% \mathrm{Cl})$ & $p$-value & Odds ratio $(95 \% \mathrm{Cl})$ & $p$-value \\
\hline Early coronary angiography & $0.71(0.58-0.88)$ & 0.002 & & \\
\hline Sex (reference = female) & $1.07(0.85-1.35)$ & 0.57 & & \\
\hline Age (per year) & $1.01(1.00-1.02)$ & 0.03 & & \\
\hline Diabetes mellitus & $1.36(1.03-1.78)$ & 0.03 & & \\
\hline Hypertension & $0.96(0.77-1.21)$ & 0.74 & & \\
\hline Arrest witnessed & $1.35(0.95-1.94)$ & 0.10 & & \\
\hline Time from arrest to BLS (per minute) & $1.07(1.03-1.11)$ & $<0.001$ & & \\
\hline Time from arrest to ROSC (per minute) & $1.03(1.02-1.04)$ & $<0.001$ & $1.12(1.06-1.19)$ & $<0.001$ \\
\hline Targeted temperature management & $1.92(1.28-2.88)$ & 0.002 & & \\
\hline Use of ACE-inhibitor or ARB & $1.97(1.43-2.70)$ & $<0.001$ & $0.20(0.04-0.91)$ & 0.04 \\
\hline Heart failure (LVEF < 45\%) & $1.57(1.09-2.26)$ & 0.02 & & \\
\hline Hypotension > 30 min & $3.19(2.43-4.18)$ & $<0.001$ & & \\
\hline Contrast used (per $10 \mathrm{~mL}$ ) & $1.05(1.03-1.08)$ & $<0.001$ & & \\
\hline Creatinine at baseline (per 1 pnt) & $1.01(1.01-1.01)$ & $<0.001$ & $1.05(1.03-1.07)$ & $<0.001$ \\
\hline Use of IABP & $1.06(0.69-1.64)$ & 0.80 & & \\
\hline \multicolumn{5}{|l|}{ First rhythm (reference $=$ VFNT) } \\
\hline PEA & $2.38(1.29-4.42)$ & 0.01 & & \\
\hline Asystole & $2.82(1.87-4.25)$ & $<0.001$ & & \\
\hline Glasgow Coma Scale < 8 & $3.27(1.63-6.54)$ & 0.001 & & \\
\hline
\end{tabular}

This figure shows the independent predictors of acute kidney injury in patients successfully resuscitated of out-of-hospital cardiac arrest. Time to return of spontaneous circulation and creatinine at baseline were independent predictors for the presence of acute kidney injury

$A C E$-inhibitor angiotensin-converting enzyme, $A R B$ angiotensin II receptor blocker, BLS basic life support, IABP intra-aortic balloon pump, LVEF left ventricular ejection fraction, $P E A$ pulseless electrical activity, ROSC return of spontaneous circulation, $V F$ ventricular fibrillation, $V T$ ventricular tachycardia

*If $p$-value of univariable analysis was $<0.1$, variables were entered in a backward multivariable analysis

and no difference in AKI between the groups [20]. Also, the present data are in line with findings from smaller retrospective cohort studies [19-22].

The reported incidence of AKI after cardiac arrest is up to $80 \%$ and depends on population and definition criteria [23]. The AKI incidence of $21.2 \%$ in our population is in line with previous results [19]. Baseline risk factors for AKI, such as an older age, might have contributed to the decision for deferral. Patients in the early angiography group more frequently had a shockable rhythm and subsequently ST-elevation on the ECG, while patients with deferred angiography were more frequently comatose and had higher levels of lactate on admission. This may reflect differences in cardiovascular performance between the groups, although the use of inotropics or vasopressors did not differ. Other baseline patient characteristics were balanced between the groups. Furthermore, in a patient-matched analysis no higher incidence of AKI was found in the early angiography group.

In accordance with previous, smaller studies, the timing of coronary angiography and amount of contrast were not associated with the development of AKI [19, 21, 22, 24]. The lack of association between early angiography and AKI occurrence in OHCA patients might partly be explained by the control of the ischemia-reperfusion phenomena as part of the post-cardiac arrest syndrome, that is clearly more beneficial than the potential risk of contrast in these patients [25]. Management of the postcardiac arrest syndrome involves intensive care support, but also coronary angiography when there are signs of persistent ischemia (ST-elevation myocardial infarction) on the ECG to preserve cardiac contractility for an effective circulation. A higher baseline creatinine level, the use of an ACEI or ARB and prolonged time to ROSC were independent predictors for AKI. The latter may reflect the intensity of ischemia-reperfusion injury after OHCA, of which the association with AKI has been noted in several studies [5,24, 26, 27]. The found protective effect of ACEI or ARB use for AKI highlights the importance of identification of patients at risk in order to reduce AKI development. Also increased creatinine on admission has been associated with development of AKI [5, 23, 24, 27].

We did not find a higher incidence of AKI with early angiography, but even a higher incidence of AKI in the deferred angiography group. Remarkably, patients without angiography developed more AKI than patients that 
did undergo angiography. This can be explained by the fact that in patients with an overall worse condition, angiography is more often delayed. This group was older and had more comorbidities. However, mortality in the delayed angiography group was not higher than in the early angiography group.

We observed an association between the development of severe AKI after OHCA and increased mortality, which is in agreement with previous reports [2, 5, 24, 2629]. Patients who developed AKI more frequently needed treatment with an inotropic or vasopressor. The optimal balance between level of perfusion and vasoactive support with regard to renal function after cardiac arrest is unknown. On the one hand, increasing mean arterial pressure improves renal function in OHCA patients, but on the other hand a higher level of vasoactive support may increase the incidence of AKI [30]. This aspect of clinical management after cardiac arrest must be studied in future research. Targeted temperature management was used as much in the early as in the deferred angiography group, however, patients who developed AKI were more frequently treated with targeted temperature management than patients without AKI. In a substudy of the TTM trial no difference in the incidence of AKI was found between temperature management at $33^{\circ} \mathrm{C}$ or $36{ }^{\circ} \mathrm{C}$ degrees [24]. Patients who received targeted temperature management in our study may have been in a worse clinical condition. Intended targeted temperature management may be a factor in the timing of coronary angiography, as in the COACT trial patients undergoing immediate angiography reached target temperature later than the delayed angiography group [20]. Although the optimal strategy for targeted temperature management is still unclear [31, 32], potential benefit from early cooling may be a reason to postpone angiography in comatose OHCA patients without STEMI [33].

One might question whether modern iodinated contrast media are nephrotoxic at all. Several studies concluded that early intervention is not harmful with regard to AKI [20-22, 26, 34]. A meta-analysis of three matched cohort studies suggested that no association between AKI and iodinated contrast exposure in critically ill patients exists at all $[19,35]$. Iodinated contrast is thought to cause acute tubular necrosis through hypoxia from renal vasoconstriction and by direct cytotoxic effects within the nephron [36-38]. Although nephrotoxicity from iodinated contrast has been well established, little is known about the pathophysiology of AKI after cardiac arrest.

The absence of augmented renal injury after early coronary angiography can be a contribution to the management of patients after OHCA without STEMI, especially in the light of the newest international guidelines [39]. At present, delayed as opposed to immediate angiography is recommended to be considered in OHCA patients without ST-elevation. In the decision process when to perform angiography, the absence of an increased AKI incidence permits both strategies, individualized according to clinical status as well as to logistical circumstances.

\section{Limitations}

To the best of our knowledge, this study is the first multicenter retrospective study to evaluate the association between timing of coronary angiography and AKI in a sizeable patient cohort. Nevertheless, several limitations of our study should be noted. First, causality of the results is difficult to determine and therefore the results should be interpreted with caution. Second, an immortal time bias exists, because patients who were included in the early or late angiography groups could not have died until their treatment. Third, data on pre-admission renal function were not available. The use of the first serum creatinine values on arrival might have underestimated prior renal function, resulting in a lower AKI incidence. However, we note that it may take 24-36 h for serum creatinine to rise after a definite renal insult [40]. Therefore, it is likely that the creatinine concentrations measured rapidly after OHCA are reasonably representative of pre-existing renal function. Furthermore, due to the retrospective study design, for several parameters, including survival and creatinine values at day 1 or 2 , some data were missing, so AKI could have been missed. AKI was defined according to AKIN criteria because we did not have enough creatinine values from day 4-7 to apply the more recent KDIGO criteria. Due to the time window of $48 \mathrm{~h}$ after admission for creatinine measurements, we have no information on the incidence of AKI post-angiography in the deferred group. However, this does not influence the conclusion that early-angiography is not associated with an increased incidence of AKI in this cohort. Fourth, potentially, there might have been other nephrotoxic agents than iodinated contrast that could have induced AKI after OHCA. Finally, we cannot exclude that performance of early angiography was influenced by factors that also could impact the occurrence of AKI.

\section{Conclusions}

Even though the incidence of AKI in OHCA patients was substantial in this large cohort study, we found that early angiography was not associated with a higher AKI incidence than a deferred angiography strategy. AKI incidence was even higher in the deferred angiography group, both before and after propensity score matching. We did not find an effect of coronary angiography on renal function when adjusted for other risk factors for 
AKI after OHCA. The present results implicate that it is safe to perform early coronary angiography with respect to the risk of developing AKI after OHCA.

\begin{abstract}
Abbreviations
ACEI: Angiotensin converting enzyme inhibitor; AKI: Acute kidney injury; AKIN: Acute Kidney Injury Network; ARB: Angiotensin II receptor blocker; CAG: Coronary angiography; Cl: Confidence interval; CRP: C-reactive protein; CT: Computerized tomography; ECG: Electrocardiogram; IQR: Interquartile range; OHCA: Out-of-hospital cardiac arrest; OR: Odds ratio; PCl: Percutaneous coronary intervention; ROSC: Return of spontaneous circulation; RRT: Renal replacement therapy; STEMI: ST-segment elevation myocardial infarction;VT/ VF: Ventricular tachycardia/ventricular fibrillation.
\end{abstract}

\section{Supplementary Information}

The online version contains supplementary material available at https://doi. org/10.1186/s13613-022-00987-w.

Additional file 1. AKI definition, Additional statistical analysis, Table S1-7, Figure S1.

\section{Acknowledgements}

Not applicable.

\section{Authors' contributions}

GNJ conceived and designed the study, performed the acquisition, interpretation of data and drafted the manuscript. JD, CAU and LSDJ contributed to the acquisition. JSL contributed to the study design and acquisition. EMS contributed to the study design, performed the statistical analysis, interpretation of data and manuscript review. DJ performed the acquisition and contributed to drafting the manuscript. TACMH, VAWMU, FRH, AB, JN, JB and PWGE contributed to the acquisition and manuscript review. PMV contributed to the statistical analysis and manuscript review. NR designed the study, performed the acquisition and manuscript review. They all agree to be accountable for all aspects of the work in ensuring that questions related to the accuracy or integrity of any part of the work are appropriately investigated and resolved. All authors read and approved the final manuscript.

\section{Funding}

This work was supported by unrestricted research grants from the Netherlands Heart Institute, Biotronik and AstraZeneca.

\section{Availability of data and materials}

The datasets used during the current study are available from the corresponding author on reasonable request.

\section{Declarations}

\section{Ethics approval and consent to participate}

The study protocol was approved by the IRB of the VU University Medical Center and ethics approval was obtained by the medical ethics committee of the VU University Medical Center, Amsterdam, the Netherlands, with approval number 2017.398. Informed consent was waived, as it was not achievable to obtain this from all patients of the large retrospective study cohort.

\section{Consent for publication}

The medical ethics committee approved that informed consent was waived, as it was not achievable to obtain this from all patients of the large retrospective study cohort. All authors consented for publication of the study.

\section{Competing interests}

Prof. Dr. van Royen reports research grants from AstraZeneca, Abbott, Philips, Biotronik and an honorarium from Medtronic. Dr. Lemkes reports grants from Biotronik and Astrazeneca, during the conduct of the study. All other authors declare no competing interests with regard to the study.

\section{Author details}

${ }^{1}$ Department of Cardiology, Amsterdam Cardiovascular Sciences, Amsterdam UMC, Vrije Universiteit Amsterdam, De Boelelaan 1117, 1081HV Amsterdam, The Netherlands. ${ }^{2}$ Department of Cardiology, Erasmus MC,'s Gravendijkwal 230, 3015CE Rotterdam, The Netherlands. ${ }^{3}$ Department of Intensive Care Medicine, Erasmus MC, Gravendijkwal 230, 3015CE Rotterdam, The Netherlands. ${ }^{4}$ Intensive Care Medicine, Maasstad Hospital, Maasstadweg 21, 3079DZ Rotterdam, The Netherlands. ${ }^{5}$ Department of Cardiology, Noordwest Ziekenhuisgroep, Wilhelminalaan 12, 1815JD Alkmaar, The Netherlands. ${ }^{6}$ Thoraxcentrum Twente, Medical Spectrum Twente, Koningsplein 1, 7512KZ Enschede, The Netherlands. ${ }^{7}$ Department of Intensive Care, Medical Spectrum Twente, Koningsplein 1, 7512KZ Enschede, The Netherlands. ${ }^{8}$ Department of Cardiology, Radboud University Medical Center, Geert Grooteplein Zuid 10, 6525GA Nijmegen, The Netherlands. ${ }^{9}$ Department of Epidemiology and Biostatistics, Amsterdam UMC, Vrije Universiteit Amsterdam, De Boelelaan 1089a, 1081 HV Amsterdam, The Netherlands. ${ }^{10}$ Department of Intensive Care Medicine, Amsterdam University Medical Centre, location VUmc, Amsterdam, The Netherlands.

Received: 23 September 2021 Accepted: 21 January 2022

Published online: 11 February 2022

\section{References}

1. Sasson C, Rogers MA, Dahl J, Kellermann AL. Predictors of survival from out-of-hospital cardiac arrest: a systematic review and meta-analysis. Circ Cardiovasc Qual Outcomes. 2010;3(1):63-81.

2. Yanta J, Guyette FX, Doshi AA, Callaway CW, Rittenberger JC, Post Cardiac Arrest $\mathrm{S}$. Renal dysfunction is common following resuscitation from outof-hospital cardiac arrest. Resuscitation. 2013;84(10):1371-4.

3. Levy EM, Viscoli CM, Horwitz RI. The effect of acute renal failure on mortality. A cohort analysis. JAMA. 1996;275(19):1489-94.

4. Sandroni C, Dell'anna AM, Tujjar O, Geri G, Cariou A, Taccone FS. Acute kidney injury after cardiac arrest: a systematic review and meta-analysis of clinical studies. Minerva Anestesiol. 2016;82(9):989-99.

5. Tujjar O, Mineo G, Dell'Anna A, Poyatos-Robles B, Donadello K, Scolletta S, et al. Acute kidney injury after cardiac arrest. Crit Care. 2015;19:169.

6. Jamme M, Ait Hamou Z, Ben Hadj Salem O, Guillemet L, Bougouin W, Pene $F$, et al. Long term renal recovery in survivors after OHCA. Resuscitation. 2019;141:144-50.

7. Mattana J, Singhal PC. Prevalence and determinants of acute renal failure following cardiopulmonary resuscitation. Arch Intern Med. 1993;153(2):235-9.

8. McCullough PA, Wolyn R, Rocher LL, Levin RN, O'Neill WW. Acute renal failure after coronary intervention: incidence, risk factors, and relationship to mortality. Am J Med. 1997;103(5):368-75.

9. Mehran R, Aymong ED, Nikolsky E, Lasic Z, lakovou I, Fahy M, et al. A simple risk score for prediction of contrast-induced nephropathy after percutaneous coronary intervention: development and initial validation. J Am Coll Cardiol. 2004:44(7):1393-9.

10. Rihal CS, Textor SC, Grill DE, Berger PB, Ting HH, Best PJ, et al. Incidence and prognostic importance of acute renal failure after percutaneous coronary intervention. Circulation. 2002;105(19):2259-64.

11. Azzalini L, Candilio L, McCullough PA, Colombo A. Current risk of contrastinduced acute kidney injury after coronary angiography and intervention: a reappraisal of the literature. Can J Cardiol. 2017:33(10):1225-8.

12. Ibanez B, James S, Agewall S, Antunes MJ, Bucciarelli-Ducci C, Bueno $\mathrm{H}$, et al. 2017 ESC guidelines for the management of acute myocardial infarction in patients presenting with ST-segment elevation: the Task Force for the management of acute myocardial infarction in patients presenting with ST-segment elevation of the European Society of Cardiology (ESC). Eur Heart J. 2018;39(2):119-77.

13. O'Gara PT, Kushner FG, Ascheim DD, Casey DE Jr, Chung MK, de Lemos JA, et al. 2013 ACCF/AHA guideline for the management of ST-elevation myocardial infarction: a report of the American College of Cardiology Foundation/American Heart Association Task Force on practice guidelines. J Am Coll Cardiol. 2013:61(4):e78-140.

14. Nolan JP, Sandroni C, Bottiger BW, Cariou A, Cronberg T, Friberg H, et al. European resuscitation council and European society of intensive 
care medicine guidelines 2021: post-resuscitation care. Resuscitation. 2021;161:220-69.

15. Welsford M, Nikolaou NI, Beygui F, Bossaert L, Ghaemmaghami C, Nonogi $\mathrm{H}$, et al. Part 5: acute coronary syndromes: 2015 international consensus on cardiopulmonary resuscitation and emergency cardiovascular care science with treatment recommendations. Circulation. 2015;132(16 Suppl 1):S146-76.

16. Mehta RL, Kellum JA, Shah SV, Molitoris BA, Ronco C, Warnock DG, et al. Acute kidney injury network: report of an initiative to improve outcomes in acute kidney injury. Crit Care. 2007;11(2):R31.

17. Bagshaw SM, George C, Bellomo R, Committe ADM. A comparison of the RIFLE and AKIN criteria for acute kidney injury in critically ill patients. Nephrol Dial Transplant. 2008;23(5):1569-74.

18. Lopes JA, Fernandes P, Jorge S, Goncalves S, Alvarez A, Costa e Silva Z, et al. Acute kidney injury in intensive care unit patients: a comparison between the RIFLE and the acute kidney injury network classifications. Crit Care. 2008;12(4):R110.

19. Petek BJ, Bravo PE, Kim F, de Boer IH, Kudenchuk PJ, Shuman WP, et al. Incidence and risk factors for postcontrast acute kidney injury in survivors of sudden cardiac arrest. Ann Emerg Med. 2016:67(4):469-76.

20. Lemkes JS, Janssens GN, van der Hoeven NW, Jewbali LSD, Dubois EA, Meuwissen $\mathrm{M}$, et al. Coronary angiography after cardiac arrest without STsegment elevation. N Engl J Med. 2019;380(15):1397-407.

21. Christ M, Auenmuller Kl, Amirie S, Brand M, Sasko BM, Trappe HJ. Acute kidney injury and renal replacement therapy in victims from out-ofhospital cardiac arrest with administration of contrast agent. Herzschrittmacherther Elektrophysiol. 2016;27(1):6-14.

22. Noc M, Fajadet J, Lassen JF, Kala P, MacCarthy P, Olivecrona GK, et al. Invasive coronary treatment strategies for out-of-hospital cardiac arrest: a consensus statement from the European association for percutaneous cardiovascular interventions (EAPCI)/stent for life (SFL) groups. Eurolntervention. 2014;10(1):31-7.

23. Kim YW, Cha KC, Cha YS, Kim OH, Jung WJ, Kim TH, et al. Shock duration after resuscitation is associated with occurrence of post-cardiac arrest acute kidney injury. J Korean Med Sci. 2015;30(6):802-7.

24. Rundgren M, Ullen S, Morgan MPG, Glover G, Cranshaw J, Al-Subaie N, et al. Renal function after out-of-hospital cardiac arrest; the influence of temperature management and coronary angiography, a post hoc study of the target temperature management trial. Crit Care. 2019;23(1):163.

25. Binks A, Nolan JP. Post-cardiac arrest syndrome. Minerva Anestesiol. 2010;76(5):362-8.

26. Chua HR, Glassford N, Bellomo R. Acute kidney injury after cardiac arrest. Resuscitation. 2012;83(6):721-7.

27. Geri G, Guillemet L, Dumas F, Charpentier J, Antona M, Lemiale V, et al. Acute kidney injury after out-of-hospital cardiac arrest: risk factors and prognosis in a large cohort. Intensive Care Med. 2015;41(7):1273-80.

28. Domanovits H, Schillinger M, Mullner M, Thoennissen J, Sterz F, Zeiner A, et al. Acute renal failure after successful cardiopulmonary resuscitation. Intensive Care Med. 2001;27(7):1194-9.

29. Hasper D, von Haehling S, Storm C, Jorres A, Schefold JC. Changes in serum creatinine in the first 24 hours after cardiac arrest indicate prognosis: an observational cohort study. Crit Care. 2009;13(5):R168.

30. Grand J, Hassager C, Winther-Jensen M, Rundgren M, Friberg H, Horn $\mathrm{J}$, et al. Mean arterial pressure during targeted temperature management and renal function after out-of-hospital cardiac arrest. J Crit Care. 2019;50:234-41.

31. Bernard SA, Smith K, Cameron P, Masci K, Taylor DM, Cooper DJ, et al. Induction of therapeutic hypothermia by paramedics after resuscitation from out-of-hospital ventricular fibrillation cardiac arrest: a randomized controlled trial. Circulation. 2010;122(7):737-42.

32. Kim F, Nichol G, Maynard C, Hallstrom A, Kudenchuk PJ, Rea T, et al. Effect of prehospital induction of mild hypothermia on survival and neurological status among adults with cardiac arrest: a randomized clinical trial. JAMA. 2014:311(1):45-52.

33. Lascarrou JB, Merdji H, Le Gouge A, Colin G, Grillet G, Girardie P, et al. Targeted temperature management for cardiac arrest with nonshockable rhythm. N Engl J Med. 2019;381(24):2327-37.

34. Joannidis M, Druml W, Forni LG, Groeneveld ABJ, Honore PM, Hoste E, et al. Prevention of acute kidney injury and protection of renal function in the intensive care unit: update 2017: expert opinion of the working group on prevention, AKI section, European society of intensive care medicine. Intensive Care Med. 2017:43(6):730-49.

35. Ehrmann S, Quartin A, Hobbs BP, Robert-Edan V, Cely C, Bell C, et al. Contrast-associated acute kidney injury in the critically ill: systematic review and Bayesian meta-analysis. Intensive Care Med. 2017;43(6):785-94.

36. Brezis M, Rosen $\mathrm{S}$. Hypoxia of the renal medulla-its implications for disease. N Engl J Med. 1995;332(10):647-55.

37. Heyman SN, Rosenberger C, Rosen S. Regional alterations in renal haemodynamics and oxygenation: a role in contrast medium-induced nephropathy. Nephrol Dial Transplant. 2005;20(Suppl 1):i6-11.

38. Persson PB, Hansell P, Liss P. Pathophysiology of contrast mediuminduced nephropathy. Kidney Int. 2005;68(1):14-22.

39. Collet JP, Thiele H, Barbato E, Barthelemy O, Bauersachs J, Bhatt DL, et al. 2020 ESC guidelines for the management of acute coronary syndromes in patients presenting without persistent ST-segment elevation. Eur Heart J. 2020.

40. Ostermann M, Joannidis M. Acute kidney injury 2016: diagnosis and diagnostic workup. Crit Care. 2016;20(1):299.

\section{Publisher's Note}

Springer Nature remains neutral with regard to jurisdictional claims in published maps and institutional affiliations.

\section{Submit your manuscript to a SpringerOpen ${ }^{\circ}$ journal and benefit from:}

- Convenient online submission

- Rigorous peer review

- Open access: articles freely available online

- High visibility within the field

- Retaining the copyright to your article

Submit your next manuscript at $\boldsymbol{\nabla}$ springeropen.com 